\title{
Strange baryons, nuclear dripline and shrinkage : A Relativistic Mean Field study
}

July 13, 2018

\author{
Bipasha Bhowmick, Abhijit Bhattacharyya and G. Gangopadhyay \\ Department of Physics, University of Calcutta \\ 92, Acharya Prafulla Chandra Road, Kolkata-700 009, India \\ email: ggphy@caluniv.ac.in
}

\begin{abstract}
Neutron and proton driplines of single- $\Lambda$ and double- $\Lambda$ hypernuclei, $\Xi^{-}$hypernuclei as well as normal nuclei are studied within a relativistic mean field approach using an extended form of the FSU Gold Lagrangian density. Hyperons are found to produce bound nuclei beyond the normal nuclear driplines. Radii are found to decrease in hypernuclei near the driplines, in line with observations in light $\Lambda$ hypernuclei near the stability valley, The inclusion of a $\Xi^{-}$introduces a much larger change in radii than one or more $\Lambda$ 's.
\end{abstract}

\section{Introduction}

The production mechanisms, spectroscopy and decay modes of hypernuclear states have been the subject of many theoretical and experimental studies 1. 2, 3, 4. Theoretical models used in studies of hypernuclei extend from nonrelativistic approaches based on One Boson Exchange models, to the relativistic mean field (RMF) approximation and quark-meson coupling models. However, the present knowledge of the $\Lambda$-N interaction in particular, and of hypernuclear systems in general, is restricted to the valley of $\beta$ stability. In view of recent advances in producing light nuclei very close to the proton and neutron drip lines using radioactive ion beams, study of ground-state properties of these nuclei has assumed importance. These exotic nuclei provide a testing ground of various theoretical models, which must explain the systematics of various properties over long chains of isotopes. Exotic nuclei on the neutron-rich side are especially important in nuclear astrophysics as they are expected to play an important role in nucleosynthesis by neutron capture. Knowledge about their structure and properties would help in the determination of astrophysical conditions for the formation of neutron-rich stable isotopes. It has been suggested [5] 
that a study of $\Lambda$ hypernuclei with a large neutron excess might also display interesting phenomena. On one hand such hypernuclei, corresponding to core nuclei which are unbound or weakly bound, are themselves of considerable theoretical interest. On the other hand, one can speculate on the possible role of neutron-rich $\Lambda$ hypernuclei in the process of nucleosynthesis.

One expects that neutron-rich hypernuclei, where a $\Lambda$ hyperon exists in the neutron-excess environment, may provide more exotic candidates than the ordinary neutron-rich nuclei, because the $\Lambda$ hyperon acts as a nuclear 'glue' in nuclei, as pointed out by Majling[5]. In fact, it was found that the core-nucleus $\alpha+d$ in ${ }_{\Lambda}^{7} \mathrm{Li}$ shrinks owing to the glue-like role of the $\Lambda[\underline{6}$. Moreover, the added $\Lambda$ can often make a system bound even if the core-nucleus is unbound. For example, ${ }_{\Lambda}^{6} \mathrm{He}$ has a bound ground state 7 though the normal nuclear core ${ }^{5} \mathrm{He}$ is unstable against neutron emission. The presence of hyperons in highdensity nuclear medium significantly affects the maximal mass of neutron stars because it softens the Equation of State (EOS). However, the baryon fraction in neutron stars is expected to depend on properties of hypernuclear potentials 8 , 9. Therefore, the study of neutron-rich hypernuclei is expected to shed light on the hypernuclear potentials in neutron-rich environment.

Several experimental attempts to produce such neutron-rich $\Lambda$ hypernuclei were carried out by double-charge exchange reactions such as (stopped $\left.K^{-}, \pi^{+}\right)$[10, 11] and $\left(\pi^{-}, K^{+}\right)$[12 on stable nuclear targets. Further experiments are planned at J-PARC facilities 13 to investigate more exotic structures of the neutron-rich hypernuclei. Various efforts have also been made on the theoretical side. For example, the relativistic Hartree-Bogoliubov model was applied to the description of $\Lambda$ hypernuclei with a large neutron excess 14. Samanta et al. used a generalized mass formula to calculate the neutron and proton drip lines of normal and lambda hypernuclei[15. In the present work the RMF theory is used to study the neutron and proton driplines of single- $\Lambda$, double- $\Lambda, \Xi^{-}$hypernuclei and compare them with the normal nuclear driplines. The FSU Gold Lagrangian density, which was extended to include hyperons [16], has been used for this purpose.

\section{Method}

In the RMF formalism [17, There are a number of standard Lagrangian densities as well as a number of different parametrizations. In the present work the FSU Gold Lagrangian density [18 has been employed. While similar in spirit to most other forces, it contains two additional non-linear meson-meson interaction terms in the Lagrangian density, whose main virtue is a softening of both the EOS of symmetric matter and the symmetry energy. As a result, the new parametrization becomes more effective in reproducing a few nuclear collective modes 18, namely the breathing mode in ${ }^{99} \mathrm{Zr}$ and ${ }^{208} \mathrm{~Pb}$, and the isovector giant dipole resonance in ${ }^{208} \mathrm{~Pb}$. The hyperon part introduced into the Lagrangian density is

$$
\mathcal{L}_{h}=\bar{\psi}_{h}\left[i \gamma_{\mu} \partial^{\mu}-M_{h}+g_{\sigma h} \sigma+g_{\sigma^{*} h} \sigma^{*}-g_{\omega h} \gamma^{\mu} \omega_{\mu}-g_{\rho h} \gamma^{\mu} \rho_{\mu}-g_{\phi h} \gamma^{\mu} \phi_{\mu}\right] \psi_{h}
$$


The explanation of different terms of the above Lagrangian density may be obtained from Bhowmick et al. [16.

In the present work neutron and proton rich hypernuclei have been studied in a RMF formalism in coordinate space in the spherical approximation. It is likely that some nuclei in the chain are deformed and also possible that the actual position of the drip line may vary slightly on inclusion of deformation. Some recent results can be found in Refs. 19 and 20. However, as our aim is to investigate possible differences in the locations of the drip lines on addition of a hyperon, we expect our results to be valid even if deformation is taken into account. A $\delta$ interaction of constant strength, i.e. $V=V_{0} \delta\left(\overrightarrow{r_{1}}-\overrightarrow{r_{2}}\right)$ has been adopted for pairing correlation. The matrix elements are calculated using the wave functions obtained for the single particle states. We choose $V_{0}=-350$ $\mathrm{MeV}$ for the strength of the delta-interaction. This value of the strength has been used by Bhattacharya et al. 21] and also by Yadav et al. 222. The valence shell has always been taken as the pairing space for both protons and neutrons.

The usual BCS equations now contain contributions from the bound states as well as the resonant continuum. For the equations involving the resonant continuum readers are referred to Sandulescu et al. 23. We have also included the effect of the width of the positive energy levels. The positive energy resonance solutions are obtained using the scattering approach. We have used the tagging approximation for odd mass nuclei. In the present work the equations have been solved using a grid of size $0.08 \mathrm{fm}$ and a box size of $20 \mathrm{fm}$ following the works of Bhattacharyaet al. 21. They had verified that the results are unaffected if a larger box size is taken. According to their calculations an increase to $22 \mathrm{fm}$ changes the total energy by less than $0.005 \%$ and radius by less than $0.05 \%$ in ${ }^{64} \mathrm{Ca}$. Besides, Zhou et al. 24, have also found that the rms radii are independent of the box size for both stable and the dripline nuclei for a box of size $>20 \mathrm{fm}$.

\section{Results}

In Fig. 1 we present the results of our calculations for the proton driplines in single- $\Lambda$, double- $\Lambda$ and $\Xi^{-}$hypernuclei and compare them with the values obtained for the normal nuclei for $N \leq 20$. The bound nuclei, with the highest number of protons for a particular $N$, have been indicated. The proton dripline does not change on addition of a single- $\Lambda$ hyperon to the normal core, except for $\mathrm{N}=8$ and 9 . In these two instances the proton dripline shifts towards more proton rich side. The inclusion of two $\Lambda$ 's shifts the p-dripline away from the stability valley in nuclei with $\mathrm{N}=2,5,7,8,9$ and 20 . The inclusion of $\Xi^{-}$has a more prominent effect on the proton dripline and shifts it even further for $\mathrm{N}=2$, $9,18,19$ while being similar to double- $\Lambda$ in its effect on nuclei with $N=8$ and 20. Interestingly, at $N=5$ inclusion of two $\Lambda$ 's, and not $\Xi^{-}$, shifts the dripline.

In Table 1, we have noted the results of proton dripline study for $20<N \leq$ 82. For brevity, only those results have been tabulated where the dripline shifts due to the inclusion of hyperon(s). For example, there is no shift of the dripline 
Table 1: Proton driplines for $\Lambda, \Lambda \Lambda$ and $\Xi^{-}$hypernuclei compared to that of the normal nuclei for $20<N<82$.

\begin{tabular}{lllll|lllll}
\hline $\mathrm{N}$ & normal & $\Lambda$ & $\Lambda \Lambda$ & $\Xi^{-}$ & $\mathrm{N}$ & normal & $\Lambda$ & $\Lambda \Lambda$ & $\Xi^{-}$ \\
\hline 27 & 28 & 28 & 28 & 30 & 56 & 52 & 52 & 52 & 54 \\
28 & 30 & 30 & 30 & 32 & 59 & 56 & 56 & 56 & 58 \\
29 & 32 & 32 & 32 & 34 & 66 & 58 & 58 & 58 & 60 \\
32 & 36 & 36 & 36 & 38 & 67 & 58 & 58 & 58 & 60 \\
33 & 36 & 38 & 38 & 38 & 68 & 60 & 60 & 60 & 62 \\
34 & 38 & 38 & 38 & 40 & 69 & 60 & 60 & 60 & 62 \\
35 & 38 & 40 & 40 & 40 & 70 & 62 & 62 & 62 & 64 \\
39 & 40 & 40 & 40 & 42 & 73 & 64 & 64 & 64 & 66 \\
40 & 40 & 42 & 42 & 44 & 75 & 66 & 66 & 66 & 68 \\
41 & 42 & 42 & 42 & 44 & 77 & 68 & 68 & 68 & 70 \\
42 & 44 & 44 & 44 & 46 & 79 & 70 & 70 & 70 & 72 \\
44 & 46 & 46 & 46 & 48 & 81 & 72 & 72 & 72 & 74 \\
45 & 48 & 48 & 48 & 50 & 82 & 72 & 72 & 72 & 74 \\
55 & 50 & 50 & 50 & 52 & & & & & \\
\hline
\end{tabular}

for $20<N<27$. It is seen from Table 1 that the inclusion of a $\Lambda$ hyperon shifts the proton dripline towards more proton rich side in a few cases $(\mathrm{N}=33,35$, and 40). The effect of two $\Lambda$ 's is identical as the effect of inclusion of a single- $\Lambda$. The inclusion of $\Xi^{-}$have much more pronounced effect shifting the proton dripline in almost all the cases tabulated here except for $\mathrm{N}=33$ and $\mathrm{N}=35$.

From Fig. 1 and Table 1 we see that the proton dripline of single $\Lambda$ hypernuclei shifts in a few light nuclei. As the number of nucleon increases the dripline merges with that for normal nuclei. This is a consequence of the weakness of the $\Lambda$-nucleon coupling effect compared to the large nucleon-nucleon contribution to the static mean field potential. In very light double- $\Lambda$ hypernuclei the effects are slightly more prominent than in single- $\Lambda$ hypernuclei. For higher masses the dripline merges with that of the normal nuclei as in the case of single- $\Lambda$ hypernuclei. The inclusion of a $\Xi^{-}$hyperon affects the proton-driplines much more prominently than both of the single and two $\Lambda$ hyperons. It can be seen from Fig. 1 that the effect of inclusion of hyperons on the proton dripline is similar irrespective of whether $\mathrm{N}$ is odd or even. Also we see that at certain places, the p-dripline does not change at all; this may be attributed to shell effects. When the drip line corresponds to a filled proton or neutron shell, in some cases the excess binding energy from addition of hyperon(s) perhaps may not play an important role in shifting the dripline. As one can see in Fig. 1, $Z=8,16$ and 20 correspond to such positions. Near drip line, $N=16$ may act as a magic number. A similar situation is observed in $N=50$ and 58 , the last being a subshell closure.

In Fig. 2 we present the results of our calculations for the neutron driplines 
Table 2: Neutron driplines for $\Lambda, \Lambda \Lambda$ and $\Xi^{-}$hypernuclei compared to that of the normal nuclei for $22<Z<50$.

\begin{tabular}{|c|c|c|c|c|}
\hline $\mathrm{Z}$ & $\begin{array}{c}\text { normal } \\
1 \mathrm{n}(2 \mathrm{n})\end{array}$ & $\begin{array}{c}\Lambda \\
1 \mathrm{n}(2 \mathrm{n})\end{array}$ & $\begin{array}{c}\Lambda \Lambda \\
1 \mathrm{n}(2 \mathrm{n})\end{array}$ & $\begin{array}{c}\Xi^{-} \\
\mathrm{n}(2 \mathrm{n})\end{array}$ \\
\hline 26 & $49(56)$ & $49(56)$ & $57(58)$ & $49(56)$ \\
27 & $49(56)$ & $49(56)$ & $57(58)$ & $49(56)$ \\
28 & $55(56)$ & $49(56)$ & $57(58)$ & $49(56)$ \\
29 & $55(56)$ & $49(56)$ & $57(58)$ & $49(56)$ \\
30 & $55(58)$ & $67(68)$ & $67(68)$ & $49(68)$ \\
31 & $63(64)$ & $67(68)$ & $67(68)$ & $67(68)$ \\
35 & $79(80)$ & $79(80)$ & $79(82)$ & $79(80)$ \\
37 & $79(80)$ & $79(80)$ & $83(84)$ & $79(86)$ \\
38 & $79(86)$ & $81(86)$ & $83(88)$ & $79(86)$ \\
39 & $79(86)$ & $81(86)$ & $83(88)$ & $79(86)$ \\
41 & $81(86)$ & $81(86)$ & $83(92)$ & $81(90)$ \\
42 & $81(90)$ & $81(90)$ & $83(92)$ & $81(108)$ \\
43 & $81(90)$ & $81(90)$ & $83(92)$ & $81(112)$ \\
44 & $81(90)$ & $81(90)$ & $83(92)$ & $81(112)$ \\
45 & $81(90)$ & $81(90)$ & $83(92)$ & $81(112)$ \\
46 & $81(90)$ & $81(90)$ & $83(92)$ & $81(112)$ \\
47 & $81(90)$ & $81(90)$ & $83(92)$ & $81(112)$ \\
48 & $81(110)$ & $81(110)$ & $83(112)$ & $81(112)$ \\
49 & $81(110)$ & $81(110)$ & $83(112)$ & $81(112)$ \\
50 & $109(110)$ & $109(110)$ & $111(114)$ & $11(112)$ \\
\hline
\end{tabular}

(for even neutron number only) in hypernuclei and compare them with that obtained for the normal nuclei for $Z \leq 22$. We see that in all the cases except for $Z=5$ and $Z=15$ the neutron dripline does not change due to inclusion of a single- $\Lambda$ hyperon. The inclusion of two $\Lambda$ 's shifts the dripline towards more neutron rich side for certain proton numbers, the effect being maximum for $\mathrm{Z}=7$ and $Z=16$. The effect of inclusion of $\Xi^{-}$is manifested only for $Z=15$ and 16 . We see that at $Z=15$, the effect of all the hyperons are visible in the shifting of neutron dripline.

In Table 2, we have noted the results of neutron dripline study for $22<$ $Z \leq 50$. One important point that should be noted is that in heavier nuclei, the neutron drip line may differ substantially for even and odd neutron numbers due to the effect of pairing. Only those results have been tabulated for which there is a change in the dripline due to the inclusion of $\Lambda, \Lambda \Lambda$, or $\Xi^{-}$. The dripline shifts towards more neutron rich side in case of $Z=29,30,31,38$ when a $\Lambda$ is present. In the neutron dripline side the inclusion of two $\Lambda$ 's is really effective in shifting the dripline and its effect shows in all the cases presented in Table 2 in the shifting of neutron drip-line. However the effect of $\Xi^{-}$on the neutron dripline is much less visible. The inclusion of $\Lambda$ binds the neutron single particle 
levels strongly, while leaving the proton single particle levels unchanged, which may be a possible reason for this. From Fig. 2 and Table 2 we see that the effect of inclusion of strange baryons, on the neutron dripline seems to be similar irrespective of whether $\mathrm{Z}$ is odd or even. Shell effects are also visible at certain neutron and proton numbers.

It is noticeable that the results of Ref. 25] for the Ca isotopes differs somewhat from our results. In that work, it was predicted that the dripline nucleus for hypernuclei of $\mathrm{Ca}$ is at $\mathrm{N}=54$ compared with that for ordinary nuclei at $\mathrm{N}=52$. However we see no shift of dripline for the Ca nucleus.

We have also investigated the change in the root mean square (r.m.s) charge radii and baryon radii due to the inclusion of a hyperon near the drip line. It is seen, that the shrinkage phenomenon in the light-mass $\Lambda$ hypernuclei is present at the dripline and this effect enhances if an additional $\Lambda$ is included. However there is a characteristic difference in the effect near the proton and the neutron driplines. At the p-dripline the r.m.s charge radius tends to decrease for $N \leq 4$, whereas at the n-dripline it is the neutron r.m.s radius that decreases for $Z \leq 7$ and also for the hypernucleus ${ }_{\Lambda}^{35} \mathrm{Si}$. However, these changes in radii are not quantitatively much significant (below $1 \%$ ).

On the other hand, the inclusion of a $\Xi^{-}$may change the radii significantly. In Figs. 3 and 4 we plot the change in mean square radii $\Delta r^{2}=\left\langle r_{H}^{2}\right\rangle-\left\langle r_{\text {core }}^{2}\right\rangle$ at the proton and the neutron driplines respectively for $\Xi^{-}$hypernuclei. Here, the suffices $H$ and core refer to hypernucleus and normal core, respectively.

From Fig. 3 it can be seen that at the p-dripline of $\Xi^{-}$hypernuclei the r.m.s charge radius decreases while the neutron radius increases. A similar effect was seen in Tan et al. 26] for nuclei away from dripline. There, a slow decrease in this effect with mass was seen. From the figure it is clear that this effect seems to die out with mass number.

Fig. 4 shows that near the neutron dripline in addition to the shrinkage in the charge radius, the neutron r.m.s radius also tends to shrink in some nuclei. The shrinkage effect increases as we go towards higher mass number and seems to saturate eventually.

These changes can be attributed to the effect of the charge and isospin of the $\Xi^{-}$. The changes in the scalar and the vector fields due to inclusion of a $\Xi^{-}$are almost identical, so that these changes cancel out each other. On the other hand, both the isovector and photon fields change significantly. The isovector field, being governed by the total isospin projection value, has opposite signs at the two driplines. This should increase the proton radius and decrease the neutron radius at the $\mathrm{n}$-dripline. However the photon field, which is more dominant, compensates for the change in proton radii, so that both the proton and neutron radii decrease at the neutron dripline. This in agreement with the work by Tan et al. 26] where, on switching off the coupling of the $\Xi^{-}$to the $\rho$ meson, only the proton radius decreased leaving the neutron radius unchanged. As the shrinkage due to the $\Xi^{-}$can be seen even at very low mass, it will be interesting to look for such changes experimentally. 


\section{Summary}

To summarize, the effect of the addition of a hyperon to a non-strange normal nucleus appears significant on both the neutron and the proton driplines, particularly in lighter mass regions. The presence of hyperons creates bound states beyond the normal dripline in many cases. Inclusion of two $\Lambda$ 's changes the neutron-dripline in many cases whereas the effect of $\Xi^{-}$on the proton dripline is more prominent. The latter effect may be attributed to the negative charge of the $\Xi^{-}$. It is seen that the inclusion of one or more $\Lambda$ makes the neutron single particle levels substantially more bound than the respective core nucleus while leaving the proton single particle levels unchanged, which may be the possible reason for the former effect. The shrinkage in size, seen in light-mass $\Lambda$ hypernuclei, is present at or near the dripline also. However the effect is different at the proton and neutron driplines. The inclusion of a $\Xi^{-}$decreases the radius more effectively than a $\Lambda$ hyperon.

\section{Acknowledgment}

This work was carried out with financial assistance of the UGC (RFSMS, DRS, UPE) and DST, Government of India.

\section{References}

[1] R. E. Chrien and C. B. Dover, Annu. Rev. Nucl. Part. Sci. 39(1989) 113.

[2] C. B. Dover, D. J. Millener, and A. Gal, Phys. Rev. C 184 (1989) 1.

[3] H. Bando, T. Motoba, and J. Zofka, Int. J. Mod. Phys. A 5 (1990) 4021.

[4] O. Hashimoto and H. Tamura, Prog. Part. Nucl. Phys. 57 (2006) 564.

[5] L. Majling, Nucl. Phys. A 585 (1995) 211c.

[6] H. Tamura et al. Phys. Rev. Lett. 84 (2000) 5963.

[7] E. Hiyama et al. Phys. Rev. C 59 (1999) 2351.

[8] S. Balberg and A. Gal, Nucl. Phys. A 625 (1997) 435.

[9] M. Baldo et al. Phys. Rev. C 58 (1998) 3688.

[10] K. Kubota et al. Nucl. Phys. A 602 (1996) 327.

[11] M. Agnello et al. Phys. Lett. B 640 (2006) 145.

[12] P.K. Saha et al. Phys. Rev. Lett. 94 (2005) 052502.

[13] A. Sakaguchi et al. , Production of Neutron-Rich $\Lambda$-Hypernuclei with the Double Charge-Exchange Reactions, Proposal for Nuclear and Particle Physics experiments at the J-PARC (2007). 
[14] D. Vretenar, W. Poschl, G. A. Lalazissis, and P. Ring, Phys. Rev. C 57 (1998) R1060.

[15] C. Samanta, P. Roy Chowdhury and D.N. Basu, J. Phys. G: Nucl. Part. Phys. 35 (2008) 065101.

[16] B. Bhowmick, A. Bhattacharyya and G. Gangopadhyay, Int. J. Mod. Phys. E 21 (2012) 1250069.

[17] P. Ring, Prog Part Nucl Phys 37 (1996) 193 and referrences therein.

[18] B.G. Todd-Rutel and J. Piekarewicz, Phys. Rev. Lett. 95 (1995) 122501.

[19] Bing-Nan Lu, En-Guang Zhao and Shan-Gui Zhou, Phys. Rev. C 84 (2011) 014328.

[20] K. Hagino et al. arXiv:1211.5871[nucl-th] (2012).

[21] Madhubrata Bhattacharya and G. Gangopadhyay, Fizika B 16 (2007) 3.

[22] H.L. Yadav, M. Kaushik and H. Toki, Int. J. Mod. Phys. E 13 (2004) 647.

[23] N. Sandulescu, L.S. Geng, H. Toki and G.C. Hillhouse, Phys. Rev. C 68 (2003) 054323.

[24] Shan-Gui Zhou, Jie Meng and P. Ring, Phys. Rev. C 68 (2003) 034323.

[25] H.F. Lu et al. ,Eur. Phys. J. A 17 (2003) 19.

[26] Y.-H. Tan, X.-H. Zhong, C.-H. Cai and P.-Z. Ning, Phys. Rev. C 70 (2004) 054306. 


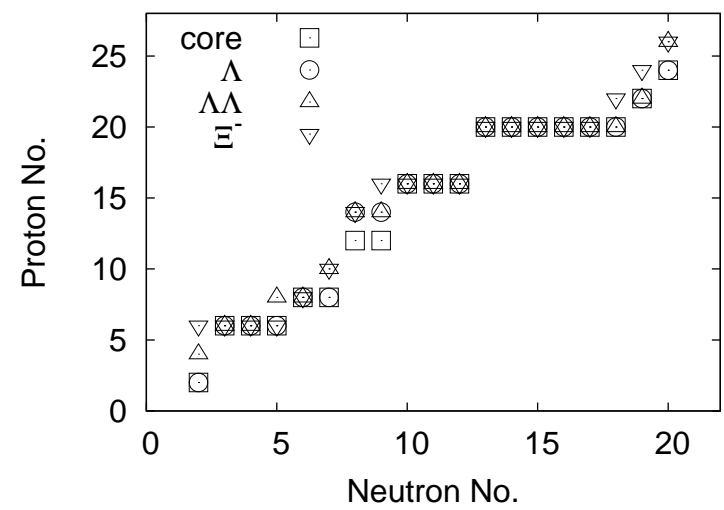

Figure 1: Proton driplines of single- $\Lambda$, double- $\Lambda, \Xi^{-}$hypernuclei as compared to that of the normal nuclei for $N \leq 20$.

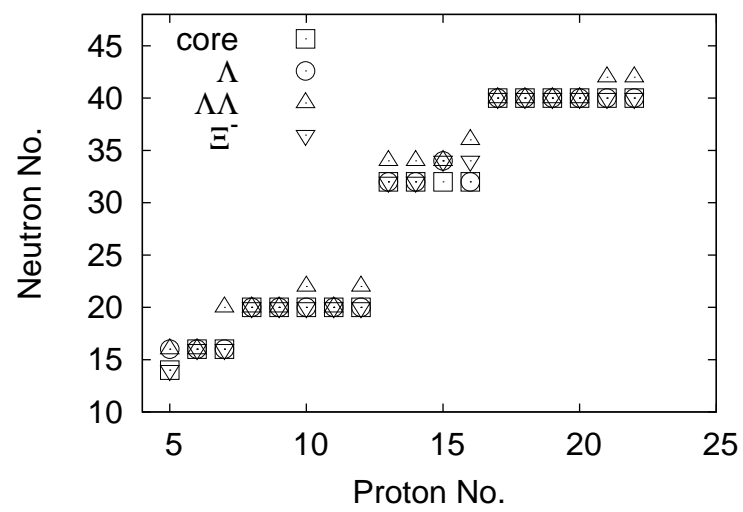

Figure 2: Neutron driplines of single- $\Lambda$, double- $\Lambda, \Xi^{-}$hypernuclei as compared to that of the normal nuclei for $Z \leq 22$. 

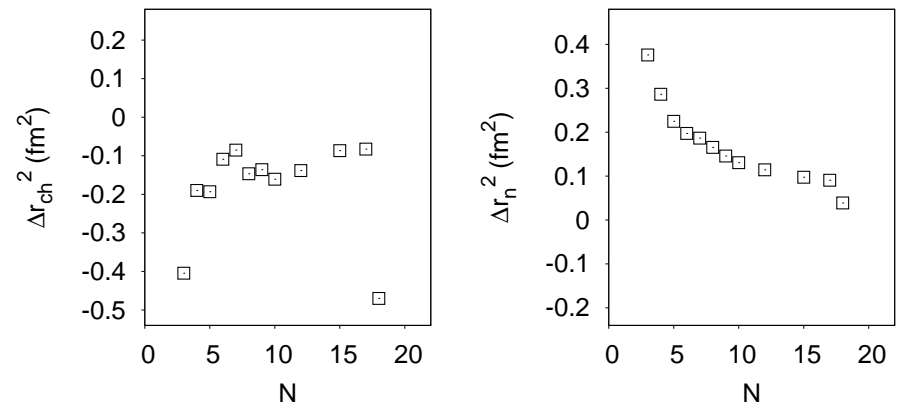

Figure 3: Change in mean square radius due to the inclusion of $\Xi^{-}$at or near the proton driplines for $N \leq 20$. see text for details.
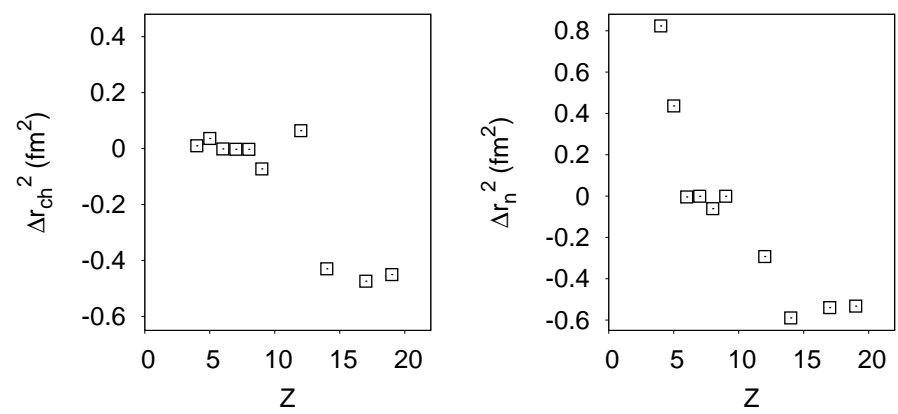

Figure 4: Change in mean square radius due to the inclusion of $\Xi^{-}$at or near the neutron driplines for $Z \leq 20$. see text for details. 